\title{
Encephalopathy after Bendamustine Treatment: A Rare Side Effect?
}

\author{
$\underline{\text { Carolina Amado }}^{1}$, Gisela Ferreira ${ }^{2}$, Fernando Silva ${ }^{2}$, Mariana Silva Leal ${ }^{1}$, Margarida Cruz $^{1}$ \\ ${ }^{1}$ Internal Medicine Department, Centro Hospitalar do Baixo Vouga, Aveiro, Portugal \\ ${ }^{2}$ Hematology Department, Centro Hospitalar do Baixo Vouga, Aveiro, Portugal
}

Received: $17 / 09 / 2020$

Accepted: $10 / 10 / 2020$

Published: 03/11/2020

How to cite this article: Amado C, Ferreira G, Silva F, Silva Leal M, Cruz M. Encelopathy after bendamustine treatment: a rare side effect? EJCRIM 2020;7: doi:10.12890/2020_002010.

Conflicts of Interests: The Authors declare that there are no competing interests.

This article is licensed under a Commons Attribution Non-Commercial 4.0 License

\section{ABSTRACT}

Bendamustine is a chemotherapeutic drug associated with frequent haematological and gastrointestinal adverse effects and, more rarely, neurological toxicity.

We present the case of a 79-year-old man with follicular lymphoma, grade 2, Ann-Arbor stage IV-A, FLIPI 4, high risk, with bulky disease and vital organ compression, treated with R-CHOP and then rituximab-bendamustine, who developed encephalopathy approximately 2 months after the last cycle. After ruling out other possible aetiologies, we assumed the patient's encephalopathy was due to a delayed bendamustine side effect, possibly aggravated by the concomitant administration of rituximab.

The authors hope to alert clinicians to this rare adverse effect, its difficult identification and the need to rule out other aetiologies.

\section{LEARNING POINTS}

- Bendamustine neurotoxicity is a rare side effect.

- Bendamustine neurotoxicity should be considered a diagnosis of exclusion in a patient with progressive encephalopathy treated with the drug.

- Drug cessation is the mainstay of treatment in cases of bendamustine-induced encephalopathy.

\section{KEYWORDS}

Follicular lymphoma, bendamustine, rituximab, neurotoxicity, encephalopathy

\section{INTRODUCTION}

Despite being an old conventional chemotherapeutic drug ${ }^{[1]}$, bendamustine is still recommended in the treatment of indolent $\mathrm{B}$-cell lymphomas, namely, symptomatic follicular lymphomas. It has a unique structure, being composed of an alkylating agent and a purinelike benzimidazole ring ${ }^{[1,2]}$, aimed at reducing the toxicity of the nitrogen mustard moiety ${ }^{[2]}$. Known adverse effects are mainly related to haematological and gastrointestinal toxicity [3], although there are a few reports of neurological toxicity ${ }^{[4,5]}$.

The authors report a case of encephalopathy after rituximab-bendamustine ( $R-B)$ treatment.

\section{CASE DESCRIPTION}

A 79-year-old man, with previous history of arterial hypertension and hypercholesterolaemia, was diagnosed with follicular lymphoma, grade 2, Ann-Arbor stage IV-A, FLIPI 4, high risk, with bulky disease and vital organ compression. He was treated with eight cycles of rituximab, cyclophosphamide, doxorubicin, vincristine and prednisone (R-CHOP), with no reported toxicities. 
At response evaluation, he showed disease progression for which he underwent second-line treatment with six cycles of R-B (of note, obinutuzumab was, at that time, not available in our hospital). The patient tolerated all six cycles relatively well. However, approximately 2 months after the last cycle, he developed progressive impairment of consciousness, associated with inability to perform activities of daily life. He was first evaluated by the psychiatry department, who raised the possibility of a depression reactive to the disease progression, and he started mirtazapine, without improvement. Soon afterwards, he started to become more disorientated and non-reactive, and thus was taken back to the emergency department. On physical examination, he was conscious, presenting slowed speech and non-attention. The remaining examination was normal, including neurological evaluation. Blood analysis showed no infection parameters, normal vitamin B12 and folate levels, negative HIV/HBV/HCV serologies, non-reactive VDRL and normal thyroid function. The patient underwent two head CT scans and then MRI of the brain which showed no abnormal enhancement, haemorrhage, ischaemia or cord compression that could account for his symptoms. Cerebrospinal studies were negative and did not show any leptomeningeal infiltration by lymphoma or infectious aetiology (bacterial, simplex herpes virus, human herpesvirus 6, JC virus, toxoplasma, CMV, EBV and fungi). The electroencephalogram (EEG) was compatible with moderate encephalopathy.

After ruling out possible aetiologies of the clinical findings, we assumed that the patient's neurological impairment was due to a delayed bendamustine side effect, possibly potentiated by concomitant administration of rituximab ${ }^{[6]}$. He was treated with high doses of intravenous methylprednisone and immunoglobulin, without much clinical improvement. One month later, he underwent a control MR, EEG and CSF analysis that showed no changes compared to the first investigations. The patient's condition progressively deteriorated and he eventually died.

\section{DISCUSSION}

The R-B protocol is being increasingly used as an option for patients diagnosed with indolent B-cell lymphomas. Although bendamustine is a generally well-tolerated drug ${ }^{[3]}$, the present case demonstrates the possibility of severe neurological sequelae related to its use.

Bendamustine has a benzimidazole ring ${ }^{[1,2]}$ that has been associated with the appearance of delayed and possibly irreversible neurotoxicity ${ }^{[4,5]}$, due to its capacity to penetrate the central nervous system ${ }^{[4]}$. Eventually, the alkylating agent, which is also part of this structure, can contribute to this toxicity once the agents have impaired consciousness ${ }^{[1]}$. The authors also believe that this neurotoxicity could be potentiated by rituximab, leading to chronic encephalopathy ${ }^{[6]}$.

Taking the last arguments into account and since the wide investigation performed could not identify any other cause for our patient's neurotoxicity, the authors suspect he developed encephalopathy secondary to bendamustine.

One limitation of this study is the lack of post-mortem evaluation of the brain which might have revealed significant findings (an autopsy was not performed due to bureaucratic problems).

The authors hope to alert clinicians to the need to consider bendamustine neurotoxicity as a possible cause of progressive encephalopathy in a patient treated with the drug, even though this is considered a rare side effect. Further studies are needed to clarify the incidence and the mechanism underlying this rare but potentially lethal toxicity.

\section{REFERENCES}

1. Munakata W, Tobinai K. The discovery and the development of bendamustine for the treatment of non-Hodgkin lymphoma. Expert Opin Drug Discov 2016;11(11):1123-1130.

2. Vidal L, Gurion R, Shargian L, Dreyling M, Gafter-Gvili A. Bendamustine for patients with indolent B cell lymphoproliferative malignancies including chronic lymphocytic leukaemia - an update meta-analysis. Br J Haematol 2019;186(2):234-242.

3. Cheson BD, Brugger W, Damaj G, Dreyling M, Kahl B, Kimby E, et al. Optimal use of bendamustine in hematologic disorders: Treatment recommendations from an international consensus panel - an update. Leuk Lymphoma 2016;57(4):766-82.

4. Cheson BD, Kroll ML. Bendamustine induced neurotoxicity. Clin Adv Hematol Oncol 2009;7(11):743-746.

5. Alhafez A, Aljitawi OS, Lin TL, Ganguly S, Abhyankar S, McGuirk JP. Bendamustine associated with irreversible ascending paralysis. Case Rep Hematol 2013;2013:931519.

6. Stone JB, De Angelis LM. Cancer treatment-induced neurotoxicity: a focus on newer treatments. Nat Rev Clin Oncol 2016;13(2):92-105. 